\title{
D ificultad para el diagnóstico clínico temprano del dengue en un área endémica y su impacto sobre el manejo médico inicial
}

\author{
Ruth Aralí Martínez-Vega ${ }^{1}$, Fredi Alexander Díaz-Q uijano ${ }^{1}$, \\ Luis Angel Villar-Centeno ${ }^{1}$. \\ Low concordance between early \\ clinical suspicion of dengue \\ and its serological confirmation
}

Background: Early detection of dengue could help to prevent its complications. The usefulness of clinical criteria for diagnosis of the disease must be ascertained. Aims: To assess the correlation between laboratory and clinical diagnosis of dengue, done during the first consultation in the emergency room. To estimate the impact of clinical diagnosis on the initial medical treatment. Patients and Methods: Patients older than 5 years with an acute febrile syndrome that consulted during the first 72 hours of disease, during 2004, at an emergency room in Bucaramanga, Colombia, were studied. Symptoms and the clinical diagnosis of the initial evaluation were registered. Paired serum samples for dengue specific ELISA-IgM test and viral isolation were obtained. The association of the initial clinical diagnosis with early symptoms, initial medical treatment and laboratory diagnosis was evaluated. Results: One hundred sixty eight patients were enrolled (54 with confirmed dengue infection). Clinical diagnosis of dengue was associated to a higher request of complete blood counts $(p=0.01$ ) and greater use of intravenous fluids ( $p=0.02$ ). However, clinical diagnosis was not correlated with the laboratory diagnosis ( $\mathrm{p}=0.15$ ). The percentage of agreement was less than would be expected by chance (Kappa $=0.1$ ). Headache was associated to the initial clinical diagnosis of dengue $(p=0.03)$, and only metrorrhagia was associated with confirmed dengue infection ( $p=0.04$ ). Conclusions: The early clinical suspicion of dengue has a low concordance with the laboratory confirmation of the disease (Rev Méd Chile 2006; 134: 1153-60).

(Key words: Dengue; Serology; Serologic tests)

\begin{abstract}
Recibido el 8 de agosto, 2005. Aceptado el 10 de marzo, 2006.
Este estudio es parte del proyecto 1102-04-12919, financiado por COLCIENCIAS y la Universidad Industrial de Santander.

${ }^{1}$ Centro de Investigaciones Epidemiológicas, Universidad Industrial de Santander, Bucaramanga, Colombia.
\end{abstract}

$\mathrm{E}$

dengue es la enfermedad ocasionada por el arbovirus con mayor morbilidad y mortalidad

Correspondencia a: Dra. Ruth Aralí Martínez V. Centro de Investigaciones Epidemiológicas, Facultad de Salud de la Universidad Industrial de Santander. Cra 32 № 29-31, 3er piso. Bucaramanga, Colombia. TeleFax: (7) 6345781. E mail: rutharam@yahoo.com en el mundo ${ }^{1}$. Aproximadamente la mitad de la población mundial vive en países donde éste es endémico y se estima que anualmente ocurren cerca de 100 millones de casos de dengue, los que originan aproximadamente 24.000 muertes $^{2,3}$. A pesar de los programas de control de vectores y del amplio conocimiento que se tiene del problema, en 
los últimos años se han presentado epidemias en múltiples áreas altamente urbanizadas de América Central y del Sur ${ }^{4}$. En Colombia, el dengue es una entidad endemo-epidémica ${ }^{5,6}$ y durante el año 2004, fue el país con más casos de dengue hemorrágico (DH) y muertes por esta causa en América ${ }^{6}$.

En los primeros días de enfermedad, el dengue se presenta como un síndrome febril agudo (SFA) inespecíico, que en la mayoría de los casos evoluciona sin complicaciones y se conoce como dengue clásico (DC). Sin embargo, una proporción variable de pacientes desarrolla $\mathrm{DH}$, síndrome que se caracteriza por sangrado espontáneo, trombocitopenia y extravasación del plasma, asociado a una mayor frecuencia de complicaciones y mortalidad ${ }^{2}$. Ello hace esencial identificar el dengue en la primera consulta, a fin de ofrecer una atención oportuna al paciente y así disminuir su morbimortalidad ${ }^{7}$.

La evaluación de pacientes con SFA compatible con dengue sugiere que la incidencia real de esta enfermedad oscila entre $35-90 \%$ en áreas endémi$\mathrm{cas}^{8-11}$. Sin embargo, en un estudio realizado en Venezuela, se sugiere que el valor predictivo positivo del diagnóstico clínico podńa llegar a ser de $100 \% 12$.

Aunque la correlación entre la presunción diagnóstica de dengue y el estado real de infección podría afectar el manejo, los estudios donde se comparan el diagnóstico presuntivo con el confirmado no han evaluado la influencia del diagnóstico clínico inicial del SFA inespecífico sobre las decisiones médicas, tales como la solicitud de un hemograma (prueba que incluye los valores de hemoglobina, hematocrito, recuentos de plaquetas y de leucocitos), y la administración de medicamentos parenterales y líquidos intravenosos ${ }^{7-11}$.

En el presente trabajo se determina la concordancia entre el diagnóstico clínico de dengue en un servicio de urgencias y el diagnóstico confirmado por laboratorio, y se estima el impacto del diagnóstico clínico de dengue sobre la evaluación y el manejo médico inicial. Asimismo, se evalúa la relación entre los síntomas tempranos y los diagnósticos señalados.

\section{MATERIALES Y MÉTODOS}

El presente trabajo es un estudio de cohorte prospectiva, realizado en el área metropolitana de Bucaramanga, Departamento de Santander (Colombia) durante el periodo enero a diciembre de 2004 en la Fundación Oftalmológica de Santander - Clínica Carlos Ardila Lulle (FOSCAL).

Se incluyeron individuos mayores de cinco años de edad con SFA (referido por el paciente como fiebre alta de instalación reciente o alguna temperatura axilar mayor de $38^{\circ} \mathrm{C}$ ) sin etiología evidente al examen físico, que consultaron en las primeras $72 \mathrm{~h}$ de enfermedad. Además de la fiebre, los pacientes debían tener dos o más de las siguientes manifestaciones: cefalea, dolor retroorbitario, mialgia, artralgia, erupción cutánea, manifestaciones hemorrágicas y leucopenia ${ }^{13}$. Los pacientes o los padres, en los menores de edad, aceptaron la inclusión en el estudio mediante la firma de un consentimiento informado.

Se excluyeron los pacientes que al momento del ingreso hubieran presentado alguna de las siguientes condiciones: 1 . Evidencia o sospecha clínica, obtenida mediante anamnesis o examen físico, de otro proceso infeccioso (faringitis, otitis, apendicitis, colecistitis, infecciones urinarias, etc) que explique de forma parcial o total la enfermedad actual. 2. Diagnóstico de diabetes, SIDA, cirrosis, enfermedades reumatológicas o tumorales, insuficiencia cardiaca o renal, o historia de ingesta aguda o crónica de corticoides. 3. Residencia en zona rural o de difícil acceso para el seguimiento.

Captación, evaluación y seguimiento. Diariamente se revisaron las historias clínicas de los pacientes que consultaron el día anterior por síndrome febril y se recolectaron los datos de teléfono, edad $\mathrm{y}$ dirección de vivienda. También se registraron los diagnósticos y conductas (realización de hemograma completo automatizado, manejo con analgésicos parenterales o infusión de líquidos intravenosos) adoptadas en la consulta inicial de urgencias por los médicos de la institución, quienes desconocían que el paciente sería incluido en el estudio.

En un primer tamizaje basado en esta información, se descartaron los individuos menores de cinco años o quienes tenían alguno de los criterios de exclusión. Los investigadores contactaron telefónicamente a los pacientes restantes y a aquellos individuos susceptibles de seguimiento, se les solicitó autorización para visitarlos.

A quienes aceptaron, se les realizó una evaluación médica en su domicilio, que incluyó la 
anamnesis y un examen físico completo. Los pacientes con alguna evidencia al examen físico de un foco infeccioso que explicara el SFA, fueron excluidos en este punto.

Posteriormente, entre las 48 y 96 primeras horas de enfermedad se tomó una muestra de sangre en tubos al vacío y mediante centrifugación (durante $10 \mathrm{~min}$ ) se obtuvo suero (agudo) para medición de anticuerpos IgM. Después del séptimo día de enfermedad, se obtuvo suero (convaleciente) para realizar una segunda prueba de IgM.

Pruebas diagnósticas. Para este estudio serológico se empleó un sistema de ensayo de captura (MACELISA) adaptado en el laboratorio de virología (Centro de Investigaciones en Enfermedades Tropicales-UIS) $^{8}$, la modificación incluida fue descrita anteriormente por Innis y cols $^{14}$. Se sensibilizaron por $18 \mathrm{~h} \mathrm{a} 4^{\circ} \mathrm{C}$, microplacas de 96 pozos con $100 \mu \mathrm{l}$ de anticuerpo anti-IgM humana (Sigma St. Louis, MO). Después de lavar 3 veces con tampón fosfato (PBS pH 7.4), se adicionaron $50 \mu \mathrm{l}$ del suero (diluido hasta una relación 1:40) a cada pozo y las placas se incubaron por $2 \mathrm{~h}$ a $37^{\circ} \mathrm{C}$. Después de 5 lavados se agregaron $50 \mu \mathrm{l}$ de antígeno tetravalente de dengue (CDC, Puerto Rico) y se mantuvo por 18 $\mathrm{h}$ a $4^{\circ} \mathrm{C}$, luego de lo cual se lavaron nuevamente y se adicionó $50 \mu \mathrm{l}$ de anticuerpo antidengue conjugado con peroxidasa (6B6C; CDC, Dengue Branch, Puerto Rico). Las placas se incubaron por una hora a $37^{\circ} \mathrm{C}$, se lavaron y luego se adicionó $100 \mu \mathrm{l}$ del sustrato (TMB; Sigma St. Louis, MO). La lectura se realizó a $450 \mathrm{~nm}$ en un lector de ELISA (Sensident Scan Merck) y el valor del punto de corte se calculó como el promedio de los valores de densidad óptica de los sueros controles negativos más 2 desviaciones estándar.

Adicionalmente, se intentó aislamiento viral en pacientes con IgM positiva en la convalecencia y en todos aquellos en los que no se pudo obtener la segunda muestra. Para el cultivo se emplearon las muestras de fase aguda que permanecieron almacenadas a $-70^{\circ} \mathrm{C}$ hasta el momento de la prueba. Con estos sueros, se infectaron células de mosquito Aedes albopictus (clon C6/36) mantenidas en medio Leibozt's (L-15. GIBCO, BRL) suplementado con suero bovino fetal (10\%), triptosa fosfato (10\%) y antibióticos (penicilina $100 \mathrm{UI} / \mathrm{ml}$; estreptomicina $100 \mu \mathrm{g} / \mathrm{ml}$ ) (1\%). Se adicionaron $100 \mu \mathrm{l}$ de suero a monocapas celulares crecidas en tubos de vidrio y luego los cultivos se centrifugaron a $2.000 \mathrm{rpm}$ por $30 \mathrm{~min}$ y a temperatura ambiente. Después se adicionó 1 mililitro del medio de cultivo suplementado con $2 \%$ de suero bovino fetal y los cultivos se incubaron a $32^{\circ} \mathrm{C}$ por 8 días. La presencia del virus se determinó por inmunofluorescencia directa usando un anticuerpo policlonal anti-dengue marcado con fluoresceína y luego el serotipo se identificó por inmunofluorescencia indirecta con anticuerpos monoclonales específicos. Los anticuerpos fueron gentilmente donados por el CDC, Dengue Branch Puerto Rico.

Definiciones de caso. La infección aguda por dengue se consideró confirmada, según la recomendación de la $\mathrm{OMS}^{13}$, cuando se evidenció seroconversión (IgM de suero agudo negativa e IgM de suero convaleciente positiva), cuadriplicación de los títulos de IgM contra dengue o aislamiento viral positivo. Se definió SFA de otra etiología (no dengue) cuando la prueba de IgM específica contra dengue en la muestra convaleciente fue negativa.

Se consideraron como casos indeterminados y no fueron incluidos en el análisis, aquellos con IgM positiva en ambos sueros sin aumento de los títulos de anticuerpos y los pacientes sin muestra de la convalecencia cuyo aislamiento viral fue negativo.

Almacenamiento y análisis. La información obtenida se registró en un formato estándar, se realizó doble digitación y se compararon las bases con el programa Epi Info. Se estimó el porcentaje de concordancia y el índice Kappa, comparando el diagnóstico realizado por el médico de urgencias y el real, definido por las pruebas serológicas o virológicas. Con la prueba de Chi cuadrado se determinaron las variables asociadas al diagnóstico clínico inicial y al diagnóstico confirmado de dengue.

Para estimar la comelación entre el diagnóstico clínico inicial y la conducta del médico tratante, se consideraron como variables dependientes la solicitud de hemogramas y la administración parenteral de medicamentos o líquidos. En el análisis se empleó el programa estadístico STATA, versión 5.0 (Stata. Corp.).

\section{RESULTADOS}

Durante el año 2004, la institución registró 10.933 consultas, con los diagnósticos de dengue (n 
$=665$ ), enfermedad viral no especificada ( $\mathrm{n}=6.932$ ) y síndrome febril no especificado ( $\mathrm{n}=3.336$ ). Los investigadores obtuvieron información de 3.755 pacientes, incluyendo 3.499 con las impresiones diagnósticas mencionadas y 256 con otros diagnósticos diferentes pero con SFA (Tabla 1). La diferencia entre el número de consultas y el número de pacientes se debe a que un mismo paciente podía haber consultado varias veces durante la misma enfermedad y a que la captación de pacientes se desarrollaba 5 días a la semana y sólo se revisaban las historias de los pacientes que asistían el día inmediatamente anterior.

De los pacientes disponibles ( $\mathrm{n}=3.755$ ), se excluyeron 3.574 pacientes, principalmente por problemas para contactarlos telefónicamente, evidencia de otra causa de enfermedad febril y porque al momento de contactarse, la enfermedad tenía más de 96 h de evolución (Tabla 1).

De un total de 181 pacientes con SFA evaluados prospectivamente (167 con muestras pareadas), se logró clasificar el diagnóstico en 168 individuos, 54 con dengue y 114 con otras enfermedades febriles. Los casos de dengue se confirmaron principalmente por seroconversión (45 casos), sin embargo, en 8 pacientes se detectó la $\operatorname{IgM}$ en suero agudo (sensibilidad de la IgM aguda $=14,8 \%$ y se evidenció el incremento de sus títulos en la fase convaleciente. Además, se logró aislar el virus (serotipo 3) en 7 de 50 pacientes con IgM positiva en la convalecencia y en el suero agudo de uno de los 14 pacientes sin segunda muestra (sensibilidad del aislamiento $=15,7 \%$ ).

De los 168 incluidos, en 29 (17,3\%) se consideró el diagnóstico de dengue en la consulta inicial de ungencias y, de ellos, sólo a 6 se les confirmó la infección. En los 139 restantes, que fueron reportados con otras impresiones diagnósticas, se detectaron los otros 48 casos de dengue. De esta forma, el diagnóstico clínico de dengue mostró una sensibilidad de 11,1\%; especificidad de 79,8\%, valor predictivo positivo de $20,7 \%$ y valor predictivo negativo de $65,5 \%$.

No hubo asociación estadísticamente significativa entre el diagnóstico recibido en urgencias y la probabilidad de tener dengue $(p=0,15)$. El porcentaje de concordancia entre el diagnóstico inicial y el real fue de $57,7 \%$, el cual es inferior al esperado por el azar $(61,7 \%)$, con un valor de Kappa de -0,1 (Tabla 2).

El diagnóstico clínico de dengue en la primera consulta estuvo estadísticamente asociado a la presencia de cefalea ( $p=0,03)$. Los otros síntomas evaluados

Tabla 1. Pacientes elegibles, excluidos e incluidos de acuerdo al diagnóstico clínico inicial

\begin{tabular}{|lccr|}
\hline Característica del paciente & \multicolumn{2}{c}{ Según el diagnóstico clínico } \\
Dengue & Otros & Total \\
\hline Con criterios de inclusión & 369 & $3.386^{*}$ & 3.755 \\
Total excluidos & 338 & 3.236 & 3.574 \\
Causas de exclusión: & & & \\
$\quad$ No contactados & & 1.820 & 1.996 \\
Asintomático & 176 & 291 & 304 \\
$\quad$ No aceptó & 13 & 42 & 47 \\
Comorbilidad & 5 & 15 & 19 \\
Sitio & 4 & 156 & 177 \\
$\quad>96$ horas de enfermedad & 21 & 322 & 400 \\
$\quad$ Otro diagnóstico tamizaje inicial & 78 & 476 & 133 \\
Evidencia al examen de otra etiología & 22 & 114 & 181 \\
Incluidos en el seguimiento & 19 & 150 & 168 \\
$\quad$ Con diagnóstico etiológico & 31 & 139 & \\
\hline
\end{tabular}

* Incluyendo 2.175 pacientes con diagnóstico de enfermedad de origen viral no especificada, 955 con fiebre sin otra especificación y 256 con otros diagnósticos.

† Pacientes a quienes no se pudo contactar telefónicamente debido a que no se encontraban en su domicilio, no correspondía el número telefónico o la línea se encontraba persistentemente ocupada o averiada.

‡ Estos pacientes manifestaron resolución de sus síntomas cuando se contactaron telefónicamente. 
Tabla 2. C oncordancia entre el diagnóstico clínico y el etiológico de los pacientes con dengue y otras causas de SFA*

\begin{tabular}{|lrc|}
\hline Etiología & \multicolumn{2}{c|}{ Diagnóstico clínico } \\
& Dengue & Otros \\
\hline Dengue confirmado & $6(20,7)$ & $48(34,5)$ \\
SFA no dengue & $23(79,3)$ & $91(65,5)$ \\
\hline
\end{tabular}

*Porcentaje de acuerdos $=(6+91) / 168=57,7 \%$; Kappa $=0,1$

no mostraron una asociación con esta impresión diagnóstica inicial ( $\mathrm{p}>0,05)$. La edad y el sexo tampoco influyeron en el diagnóstico dado (Tabla 3).

$\mathrm{Al}$ evaluar el manejo médico, la solicitud de un hemograma fue más frecuente cuando el diagnóstico clínico inicial fue dengue $(p=0,01)$. El uso de líquidos intravenosos (LIV) como terapia inicial, también se asoció a esta impresión diagnóstica ( $\mathrm{p}$ $=0,02$ ). No se hallaron diferencias en el uso de otros medicamentos por vía intramuscular (IM) e intravenosa (IV), tampoco en la prescripción de acetaminofen (Tabla 4).
Al evaluar la sintomatología de los pacientes, de acuerdo a los resultados del laboratorio, la metrorragia fue el único síntoma que se asoció significativamente al diagnóstico confirmado de dengue. Los otros síntomas generales y hemorrágicos evaluados no mostraron diferencias estadísticamente significativas (Tabla 5).

\section{Discusión}

El espectro clínico del dengue es muy amplio, frecuentemente se presenta con cefalea, dolor retro-ocular, osteomialgias, náuseas, odinofagia, vómito, debilidad y exantema ${ }^{1,7,8,13}$. En algunas ocasiones, este cuadro clínico también se acompaña de diarrea y síntomas respiratorios ${ }^{1,13}$. Esto hace difícil diferenciar clínicamente el dengue de otras entidades que se presentan tempranamente como un SFA inespecífico, tales como, influenza, rubéola, gastroenteritis, fiebre tifoidea, letospirosis, entre otras ${ }^{1,12,15-17 .}$

El hemograma es un recurso muy importante en la evaluación inicial y en el seguimiento del dengue porque permite evaluar el recuento plaque-

Tabla 3. C aracterísticas de los pacientes según el diagnóstico clínico inicial

\begin{tabular}{|lccc|}
\hline Característica & \multicolumn{2}{c}{ Impresión diagnóstica inicial } & Valor $\mathrm{p}^{*}$ \\
\hline Género masculino - No (\%) & $11(37,9)$ & Otros $(\mathrm{n}=139)$ & \\
Edad <15 años & $8(27,6)$ & $65(46,8)$ & 0,38 \\
Cefalea & $29(100)$ & $49(35,3)$ & 0,43 \\
Dolor retroocular & $15(51,7)$ & $120(86,3)$ & 0,03 \\
Mialgias & $27(93,1)$ & $75(54)$ & 0,83 \\
Artralgias & $21(72,4)$ & $115(82,7)$ & 0,16 \\
Tos & $15(51,7)$ & $98(70,5)$ & 0,84 \\
Rinorrea & $16(55,2)$ & $76(54,7)$ & 0,77 \\
Hiperalgesia & $5(17,2)$ & $73(52,5)$ & 0,79 \\
Exantema & $8(27,6)$ & $30(21,6)$ & 0,60 \\
Prurito & $3(10,3)$ & $22(15,8)$ & 0,13 \\
Náuseas & $20(69)$ & $20(14,4)$ & 0,56 \\
Vómito & $8(27,6)$ & $95(68,4)$ & 0,95 \\
Dolor abdominal & $16(55,2)$ & $41(29,5)$ & 0,84 \\
Mareo & $24(82,8)$ & $83(59,7)$ & 0,65 \\
Sangrado espontáneo & $5(17,2)$ & $90(54,8)$ & 0,06 \\
$\quad$ Gingivorragia & $1(3,5)$ & $10(7,2)$ & 0,08 \\
$\quad$ Epistaxis & 0 & $2(1,4)$ & 0,46 \\
$\quad$ Metrorragia & $1 / 18(5,6)$ & $4(2,9)$ & 0,36 \\
\hline
\end{tabular}

* Estimado por la prueba de $\mathrm{Chi}^{2}$. 
Tabla 4. Conductas iniciales de los médicos tratantes

\begin{tabular}{|lcccc|}
\hline & \multicolumn{2}{c}{ Diagnóstico clínico de urgencias } & RR & Valor $\mathrm{p}$ \\
Congue $(\mathrm{n}=29)$ & Otro $(\mathrm{n}=139)$ & & \\
\hline Hemograma - No (\%) & $16(55,2)$ & $42(30,2)$ & 1,83 & 0,01 \\
Medicamento IM* & $21(72,4)$ & $87(62,6)$ & 1,16 & 0,32 \\
Medicamento IV & $3(10,3)$ & $5(3,6)$ & 2,88 & 0,12 \\
Medicamentos parenterales & $23(79,3)$ & $88(63,3)$ & 1,25 & 0,1 \\
Diclofenaco IM & $17(58,6)$ & $69(49,6)$ & 1,18 & 0,38 \\
Dipirona IM & $7(24,1)$ & $26(18,7)$ & 1,29 & 0,50 \\
Dipirona IV & $1(3,4)$ & $2(1,4)$ & 2,4 & 0,461 \\
Acetaminofeno & $27(93,1)$ & $129(93,5)$ & 1,0 & 0,95 \\
LIV & $2(6,9)$ & $1(0,7)$ & 9,59 & 0,02 \\
\hline
\end{tabular}

*IM: Intramuscular; IV: Intravenoso; LIV: Líquidos intravenosos.

Tabla 5. C aracterísticas de los pacientes según el diagnóstico de laboratorio

\begin{tabular}{|lcrc|}
\hline Característica & \multicolumn{2}{c}{ Diagnóstico confirmado } & Valor $\mathrm{p}$ \\
\hline Género masculino - No (\%) & Otros $(\mathrm{n}=114)$ & \\
Edad <15 años & $25(46,3)$ & $51(44,7)$ & 0,85 \\
Cefalea & $17(31,5)$ & $40(35,1)$ & 0,64 \\
Dolor retroocular & $46(85,2)$ & $103(90,4)$ & 0,32 \\
Mialgias & $32(59,3)$ & $58(50,9)$ & 0,31 \\
Artralgias & $44(81,5)$ & $98(86)$ & 0,45 \\
Tos & $37(68,5)$ & $82(71,9)$ & 0,65 \\
Rinorrea & $27(50)$ & $64(56,1)$ & 0,46 \\
Hiperalgesia & $24(44,4)$ & $65(57)$ & 0,13 \\
Exantema & $24(44,4)$ & $66(57,9)$ & 0,10 \\
Prurito & $11(20,4)$ & $19(16,7)$ & 0,56 \\
Náuseas & $8(14,8)$ & $15(13,2)$ & 0,77 \\
Vómito & $40(74,1)$ & $75(65,8)$ & 0,28 \\
Dolor abdominal & $18(33,3)$ & $31(27,2)$ & 0,41 \\
Diarrea & $33(61,1)$ & $66(57,9)$ & 0,69 \\
Mareo & $20(37)$ & $39(34,2)$ & 0,72 \\
Sangrado espontáneo & $39(72,2)$ & $75(65,8)$ & 0,40 \\
$\quad$ Gingivorragia & $3(0,06)$ & $12(0,11)$ & 0,29 \\
$\quad$ Epistaxis & $1(0,02)$ & $2(0,02)$ & 0,96 \\
$\quad$ Metrorragia & $0(0)$ & $4(0,04)$ & 0,16 \\
\end{tabular}

tario y el hematocrito, ambos parámetros de severidad de la enfermedad ${ }^{1}$. Por otra parte, el principio fundamental en el tratamiento del dengue es la hidratación, ya sea por vía oral o intraveno$\mathrm{sa}^{3,18}$. En este estudio se observó que la impresión clínica de dengue se asoció tanto a la solicitud de hemogramas como a la utilización de líquidos intravenosos. Lo anterior soporta la necesidad de una adecuada correlación entre la sospecha clínica y el estado real de enfermedad, para optimizar los recursos asistenciales.
Sin embargo, se evidenció que el diagnóstico clínico inicial, que orientó decisiones importantes en la evaluación y tratamiento, no guardó relación con el diagnóstico real confirmado por el laboratorio. Lo anterior podría reflejar la inespecificidad del cuadro clínico ocasionado por el dengue en las primeras horas de enfermedad. Esta discordancia se hace especialmente notoria si se tiene en cuenta que durante el periodo del estudio, Colombia fue el país de América que reportó más casos de $\mathrm{DH}^{19}$ y que en los últimos años, se han presentado 
entre 113,4 y 268,7 casos de dengue por 100.000 habitantes en la región donde se desarrolló el presente estudio ${ }^{20}$. Con esta condición de área endémica, se esperaría que el diagnóstico clínico inicial tuviera un valor predictivo positivo superior al evidenciado en el presente estudio.

Es importante considerar que en Colombia, una vez que se hace un diagnóstico clínico de dengue, es obligatorio, por norma del Ministerio de Protección Social, reportar el caso a través de una ficha epidemiológica unificada, que incluye datos demográficos, clínicos y de laboratorio ${ }^{21}$. Sin embargo, se ha descrito que existe un subregistro de los casos sospechosos de dengue (incluyendo $\mathrm{DH})^{22}$. A manera de hipótesis, es probable que el tiempo requerido para llenar la ficha, que debe hacerse durante la consulta médica, sea un factor que influya negativamente sobre la notificación de los casos y la utilización del diagnóstico en las historias clínicas.

En general, la notable dificultad para identificar los casos de dengue en las primeras horas de enfermedad, podría explicarse tanto por la presentación clínica inespecífica, como por los problemas atribuibles al proceso de notificación. Lo anterior reafirma la necesidad de encontrar síntomas, signos o hallazgos de laboratorio que ayuden a mejorar el diagnóstico clínico de dengue y establecer reglas de decisión clínica para su identificación y manejo, especialmente en el periodo temprano de la enfermedad cuando las pruebas serológicas son poco sensibles ${ }^{23}$.

Al igual que otros estudios, no encontramos diferencias significativas entre los pacientes con dengue y el grupo de no dengue al evaluar síntomas generales como cefalea, artralgias y mialgias; respiratorios, como rinorrea y tos; gastrointestinales, como náuseas, vómito y dolor

\section{REFERENCIAS}

1. Guzman MG, Kouri G. Dengue and dengue hemorrhagic fever in the Americas: lessons and challenges. J Clin Virol 2003; 27: 1-13.

2. Guha-Sapir D, Schimmer B. Dengue fever: new paradigms for a changing epidemiology. Emerg Themes Epidemiol 2005; 2: 1.

3. Stephenson JR. The problem with dengue. Trans R Soc Trop Med Hyg 2005; 99: 643-6.

4. Siqueira JB Jr, Martew CM, Coelho GE, Simplicio AC, HATCH DL Dengue and dengue hemomhagic fever, abdominal; y exantema, entre otros ${ }^{10-11}$. Únicamente, la metrorragia estuvo estadísticamente asociada al diagnóstico de dengue, aunque esta manifestación mostró una muy baja sensibilidad para la detección temprana de la enfermedad. La combinación de las manifestaciones hemorrágicas con los otros síntomas, no aumentó la fuerza de la asociación con la infección por dengue (datos no mostrados). En contraste con estos resultados, en algunos reportes se ha descrito que el dolor abdominal y el vómito se asocian al diagnóstico confirmado de dengue, mientras que la odinofagia, la tos y la rinorrea, se relacionan con otras causas de SFA ${ }^{9,12}$.

En este estudio se evidenciaron problemas para contactar a un gran número de pacientes lo que sumado a la necesidad de captarlos tempranamente, llevaron a que sólo se pudiera estudiar una pequeña proporción de los casos febriles sin etiología aparente. Pese a ello, consideramos que estas circunstancias no introdujeron sesgos pues es poco plausible que estén relacionadas a la presentación clínica o a la etiología de la enfermedad.

En resumen, los hallazgos del presente trabajo sugieren que el diagnóstico clínico reportado en la primera evaluación, es un determinante de la utilización de pruebas complementarias y del tratamiento inicial, sin embargo, éste no se comelaciona con el estado real de infección. Los síntomas evaluados no parecen ayudar a esclarecer el diagnóstico tempranamente en esta población. Consideramos que deben desarrollarse estudios adicionales buscando herramientas para la identificación oportuna del dengue, cuya aplicación podnía disminuir la morbimortalidad y ayudanía a distribuir eficientemente los recursos diagnósticos y terapéuticos, especialmente en las áreas endemo-epidémicas.

Brazil, 1981-2002. Emerg Infect Dis 2005; 11: 48-53.

5. SIVIGILA. Enfermedades de notificación obligatoria. Informe primer semestre de 2003. Boletín Epidemiológico Semanal. Semana epidemiológica No. 26. Junio 22-28 de 2003. www.col.opsoms.org/sivigila/2003/BOLE26_03.pdf

6. Pan American Health Organization. 2004: Number of Reported Cases of Dengue \& Dengue Hemorrhagic Fever (DHF), Region of the Americas (by country and subregion). last update: 1 June 2005. www.ops-oms.org/English/AD/DPC/ $\mathrm{CD} /$ dengue-cases-2004.htm 
7. Narayanan M, Aravind MA, Thilothammal N, Prema $R$, Sargunam CS, Ramamurty N. Dengue fever epidemic in Chennai-a study of clinical profile and outcome. Indian Pediatrics 2002; 39: 1027-33.

8. Martínez RA, Díaz FA, Vimar LA. Evaluación de la definición clínica de dengue sugerida por la OMS. Biomédica 2005; 25: 412-6.

9. Kalayanarooj S, Vaughn DW, Nimmannitya S, Green S, Suntayakorn S, Kunentrasai N et al. Early Clinical and Laboratory Indicators of Acute Dengue Illness. J Infect Dis 1997; 176: 313-21.

10. Phuong CX, Nhan NT, Kneen R, Thuy PT, van Thien C, NGa NT ET al. Clinical diagnosis and assessment of severity of confirmed dengue infections in Vietnamese children: is the world health organization classification system helpful? Am J Trop Med Hyg 2004; 70: 172-9.

11. Rodrígues MB, Freire HB, Correa PR, Mendonca ML, Silva MR, Franca EB. Is it possible to identify dengue in children on the basis of Ministry of Health criteria for suspected dengue cases? J Pediatr (Rio J) 2005; 81: 209-15.

12. Costa de León L, Estevez J, Monsalve de CastiLo F, CALLjas D, EchevarRía JM. Laboratory diagnosis of patients with exanthematic or febrile syndromes occurring in the Zulia State, Venezuela, during 1998. Rev Méd Chile 2004; 132: 1078-84.

13. WHO. Clinical diagnosis. In: World Health Organization. Dengue haemorrhagic fever: diagnosis, treatment, prevention and control. $2^{\text {nd }}$ edition. Geneva; 1997. Pag. 12-23.

14. InNIs BL, Nisaiak A, Nimmanntiya S, Kusaitrdchariya $S$, CHONGSWASDI V, SuntaYAKoRn S ET al An enzyme-linked immunosorbent assay to characterize dengue infections where dengue and Japanese encephalitis cocirculate. Am J Trop Med Hyg 1989; 40: 418-27.

15. Watt G, Jonsgakul $K$, Chouriyagune $C$, Paris $R$. Diferentiating Dengue virus infection from scrub
Typhus in Thai adults with fever. Am Trop Med Hyg 2003; 68: 536-8.

16. LevetT PN, Branc L, EdwaRds CN. Detection of Dengue infection in patient investigated for leptospirosis in Barbados. Am J Trop Med Hyg 2000; 62: 112-4.

17. Silarug N, Foy HM, Kupradinon S, Rojanasuphot S, Nisalak A, Pongsuwant Y. Epidemic of fever of unknown origin in rural Thailand, caused by influenza A (H1N1) an dengue fever. Southeast Asian J Trop Med Public Health 1990; 21: 61-7.

18. Harris E, Pérez L, Phares CR, Pérez M, Idiaquez W, Rocha J ET AL. Fluid Intake and Decreased Risk for Hospitalization for Dengue Fever, Nicaragua. Emerg Infect Dis 2003; 9: 1003-6.

19. Pan American Health Organization. 2004: Number of Reported Cases of Dengue \& Dengue Hemonhagic Fever (DHF), Region of the Americas (by country and subregion). Disponible en: www.paho.org/English/AD/DPC/CD/dengue-cases-2004.htm

20. Ministerio de la Protección Social, Instituto Nacional de Salud. Situación epidemiológica de las enfermedades transmitidas por vectores 20032004. Boletín Epidemiológico Semanal, SIVIGILA. Semana epidemiológica № 08. Febrero 22-28 de 2004. Disponible en: www.col.ops-oms.org/sivigila/2004/bole08 04.htm

21. Ministerio de Salud. República de Colombia. Guía de Atención del Dengue. www.metrosalud.gov.co/Paginas/Protocolos/MinSalud/guias/33-DENGUE.htm

22. Camacho T, de la Hoz F, Cárdenas V, Sánchez C, de CALDERÓn L, PÉrez L eT al. Incomplete surveillance of a dengue-2 epidemic in Ibague, Colombia, 1995-1997. Biomedica 2004; 24: 174-82.

23. Díaz-Quijano FA, Martínez-Vega RA, Ocazionez RE, Vilar-Centeno LA. Evaluación de la prueba de IgM en suero agudo para el diagnóstico del dengue en un área endémica. Enferm Infecc Microbiol Clin. In press.

\section{Agradecimientos}

Por su colaboración en la realización de las pruebas diagnósticas, agradecemos a la Dra. Raquel Elvira Ocazionez, los bacteriólogos Fabián Cortés y Sergio Gómez, del Centro de Investigaciones en Enfermedades Tropicales (CINTROP), y a Karol Torres del Laboratorio Clínico de la Universidad Industrial de Santander. Agradecemos también al personal de la oficina de Estadística de la FOSCAL, por su valiosa ayuda en la obtención de la información consignada en las historias clínicas. 